\title{
Sarkozysme et populisme. Approche logométrique du discours de Nicolas Sarkozy (2007-2012)
}

Sarkozysm and populism. A Logometric approach to Nicolas Sarkozy's discourse (2007-2012)

Sarkozysme et populisme. Approche logométrique du discours de Nicolas

Sarkozy (2007-2012)

\section{Damon Mayaffre}

\section{OpenEdition}

\section{Journals}

Édition électronique

URL : https://journals.openedition.org/mots/21489

DOI : $10.4000 /$ mots. 21489

ISSN : 1960-6001

Éditeur

ENS Éditions

\section{Édition imprimée}

Date de publication : 16 décembre 2013

Pagination : 73-87

ISBN : 978-2-84788-527-9

ISSN : 0243-6450

\section{Référence électronique}

Damon Mayaffre, «Sarkozysme et populisme. Approche logométrique du discours de Nicolas Sarkozy (2007-2012) », Mots. Les langages du politique [En ligne], 103 | 2013, mis en ligne le 16 décembre 2015, consulté le 23 avril 2022. URL : http://journals.openedition.org/mots/21489 ; DOI : https://doi.org/ $10.4000 /$ mots. 21489 


\section{Sarkozysme et populisme. Approche logométrique du discours de Nicolas Sarkozy (2007-2012)}

Nicolas Sarkozy et le sarkozysme ne sont pas des épiphénomènes de la vie politique française contemporaine. D'abord parce que l'accession d'un homme à l'Élysée sous une république présidentielle comme l'est devenue la Ve République ne peut être tenue pour une manifestation politique mineure. Ensuite parce que N. Sarkozy ne domina pas la vie française, de manière éphémère, durant seulement un quinquennat, mais pendant une décennie entière si l'on veut bien considérer le poids politique et médiatique que le ministre de l'Intérieur, le ministre de l'Économie et d'État, le président de l'UMP eut face à un Jacques Chirac sénescent dès 2002. Enfin et surtout car ce que N. Sarkozy incarna semble devoir se prolonger dans le temps après la défaite de 2012, à en croire la figure tutélaire qu'il représente déjà pour la droite française, et se comprendre dans l'espace occidental si l'on veut bien rapprocher la néo-droite française de la néo-droite américaine et européenne, notamment berlusconienne en Italie, au détour des années deux mille ${ }^{1}$.

Mais qu'incarne au fond N. Sarkozy? Un programme politique bien sûr, qu'on ne saurait traiter ici, tendu vers la réduction de l'État-Providence jugé comme historiquement dépassé, et la promotion de la responsabilité individuelle sous la double forme de la récompense de la réussite et de la sanction des déviances. Un mode de gouvernance surtout et un discours plus encore - des mots, des phrases, une rhétorique - qui font rupture avec le discours traditionnel de la droite républicaine (gaullienne, giscardienne, chiraquienne).

Le sarkozysme est avant tout un discours ou une expression politique à l'orée du $21^{\mathrm{e}}$ siècle, et l'étude systématique et comparée du corpus Sarkozy (2007-2012) que nous avons entreprise montre qu'il s'agit d'un discours politique plein, riche, idéologiquement structuré sur plusieurs années malgré

1. Pierre Musso a systématiquement montré la ressemblance du sarkozysme et du berlusconisme, jusqu'à parler de «sarkoberlusconisme» : voir Musso, 2011.

2. C'est l'affirmation principale d'Esprit : voir Qu'est-ce que le sarkozysme?, n³39, novembre 2007.

Université de Nice Sophia Antipolis, BCL (UMR 7320, CNRS)

DamonMayaffre@wanadoo.fr

Mots. Les langages du politique $\mathrm{n}^{\circ} 103$ novembre $2013 \bullet 73$ 
d'inévitables vicissitudes conjoncturelles; discours nouveau, donc, au sommet de l'État si on veut bien le comparer, dissensuel ou clivant - là où le discours présidentiel est habituellement consensuel -, discours volontariste, hyperbolique, compassionnel, etc. 3 Nous ne prétendons donc pas ici épuiser toutes ses facettes et sa complexité sans égale sous la Cinquième République mais seulement évoquer une de ses dimensions majeures - de rupture, précisément, avec le passé élyséen - qui fait du discours de N. Sarkozy un discours présidentiel aux accents populistes 4 .

Le populisme est un concept de science politique difficile à manier5 qui peut recouvrir des réalités multiformes, tant par ses déclinaisons géographiques (en Europe ou en Amérique latine) qu'historiques (dans les années trente aussi bien que dans les années quatre-vingt-dix). Ses analystes mettent en garde contre une réduction du concept aux seules formes extrêmes et césaristes : le populisme, tout au contraire, est un avatar de l'âge démocratique et peut affecter les leaders des démocraties parlementaires, présidentielles ou monarchiques. Yves Mény et Yves Surel n’hésitent pas ainsi à pointer ce qu'il y a de «populiste» chez Thatcher, Blair, Chirac, Berlusconi, etc. (Mény, Surel, 2000).

Pierre-André Taguieff, quant à lui, met en garde contre la vision du populisme comme un programme politique systématique et radical pour nous orienter sur la voie plus ténue, mais non moins essentielle, d'une réalité stylistique ou discursive :

[...] le populisme, oscillant entre l'autoritarisme et l'hyperdémocratisme non moins qu'entre le conservatisme et le progressisme réformiste [...] ne p[eu]t être considéré ni comme une idéologie politique, ni comme un type de régime, mais comme un style politique, fondé sur le recours systématique à la rhétorique de l'appel au peuple et la mise en œuvre d'un mode de légitimation de type charismatique, le plus propre à valoriser le «changement». (Taguieff, 2007, p. 9; nous soulignons)

Le populisme serait donc avant tout un discours; comme le sarkozysme. Et le discours de N. Sarkozy semble reprendre, voire systématiser, les procédés discursifs populistes tels que la littérature les décrit : (i) sacralisation, dans les mots, du peuple et communion avec lui ; (ii) charisma ou construction, par le discours, de l'image du chef susceptible de guider voire d'incarner ledit peuple; (iii) dénonciation des corps intermédiaires, des élites qualifiées de bien-pensantes, de la pensée dénoncée comme «unique » que l'on opposera au bon sens populaire, pour proposer un modèle de dialogue et de gouvernement, sans médiation, entre le peuple et le chef charismatique. Ce sont

3. Pour l'ensemble de ces conclusions, voir Mayaffre, $2012 \mathrm{a}$.

4. Pour une comparaison du discours de N. Sarkozy avec celui de De Gaulle, Pompidou, Giscard d'Estaing, Mitterrand et Chirac, voir Mayaffre, 2012b.

5. Certains auteurs, par prudence, vont jusqu'à refuser de l'utiliser, le jugeant à la fois trop flou, trop polémique et trop dévalorisant dans son acception courante. Pour une réflexion sur le sujet, voir par exemple Collovald, 2004. 
ces trois dimensions nécessaires du parler populiste que cette contribution essaye d'établir, traitement statistique à l'appui, dans la bouche de N. Sarkozy.

L'étude entreprise repose en effet sur un traitement logométrique des discours de N. Sarkozy en tant que président (2007-2012) et en tant que candidat (2007 et 2012) ; discours qui ont été systématiquement comparés à des discours comparables, c'est-à-dire émis dans des conditions d'énonciation identiques ${ }^{6}$. Selon la morphologie du mot logométrie, ce traitement consiste à prendre la mesure du discours dans ses dimensions qualitatives et quantitatives : le traitement statistique des unités du discours (calcul du vocabulaire spécifique, calcul des cooccurrences, distances intertextuelles, etc.) et le retour systématique au texte pour une lecture traditionnelle (lecture et navigation hypertextuelles plein texte, lecture de concordances, recherche de passages par mot clef, etc.) se combinent. En tant que dépassement du traitement lexicométrique, l'analyse logométrique porte son attention sur toutes les unités du discours, de la lettre aux isotopies, et pas seulement aux lexies ou mots graphiques : en effet, parfois, l'idéologie transpire d'un enchaînement syntaxique surutilisé ou d'un code grammatical suremployé autant que d'un lexique ramené à une graphie. Enfin, les objectifs de ce traitement sont désormais bien compris dans le cadre du tournant herméneutique de l'analyse du discours et des sciences humaines et sociales : moins que "prouver» le sens des textes, il s'agit de contrôler l'interprétation. Le sens n'est qu'interprétation, et l'enjeu de l'analyse du discours est d'objectiver des parcours interprétatifs, ou de proposer des parcours de lecture identifiables, reproductibles, heuristiques?.

En l'occurrence, lorsqu'on compare de manière systématique les discours de N. Sarkozy à ceux de ses prédécesseurs à l'Élysée, les premières sorties machines sont intrigantes ou heuristiques. Avant quelques mots-pleins prévisibles, comme crise ou travail que N. Sarkozy surutilise, nous trouvons une série d'unités discursives très prégnantes et non intuitives : le pronom démonstratif neutre, le mot graphique on, le syntagme verbal je veux, l'enchaînement syntaxique [pronom + adverbe + verbe], le signe de ponctuation interrogatif. C'est l'interprétation de ces phénomènes discursifs non obvies et récurrents

6. Deux types de discours sarkozystes ont donc été saisis et étudiés. Le corpus présidentiel composé de l'exhaustivité des prestations télévisées (vœux, interview du 14 juillet, Journal de 20 heures, etc.) et une part des discours grand public (discours de Grenoble, de Toulon, etc.) de N. Sarkozy durant son quinquennat : ces 51 discours (283248 occ.) ont alors été comparés aux discours de De Gaulle, Pompidou, Giscard, Mitterrand et Chirac durant leur présidence (524 discours, 2522876 occ.). Par ailleurs, les discours des campagnes du candidat Sarkozy en 2007 et 2012 (63 discours, 345566 occ.) ont été comparés aux discours des autres candidats des élections présidentielles (96 discours, 598045 occ.).

7. Voir Guilhaumou, 2006 ou Rastier, 2011. De notre côté, nous avons essayé d'établir la logométrie dans notre thèse HDR : "Vers une herméneutique numérique. Corpus textuels, logométrie et discours politique», Université de Nice, 2010, Hal : http://tel.archives-ouvertes. $\mathrm{fr} / \mathrm{tel}-00655380 / \mathrm{fr} /$. 
chez N. Sarkozy qui nous mène à la définition d'un discours populiste, car tous - du moins ceux que l'on vient de nommer - semblent converger vers la mise en forme d'un discours populaire, plébéien ou plébiscitaire qui vise moins à convaincre par l'argumentaire ou par le programme que par l'appel au peuple.

\section{Le tribun : communier avec vox populi}

\section{Le ça et la doxa}

Nous avons comparé une cinquantaine de discours de Nicolas Sarkozy à 500 discours prononcés par de Gaulle, Pompidou, Giscard, Mitterrand et Chirac durant leur présidence respective, et prononcés dans des conditions équivalentes. Et s'il fallait ne retenir qu'une seule caractéristique statistique du discours de N. Sarkozy, entre toutes, ce serait la surutilisation (i) du pronom démonstratif, (ii) particulièrement du pronom démonstratif neutre, (iii) massivement sous sa forme vulgaire ou populaire «ça », et (iv) de préférence en position sujet (versus objet) (figure 1).

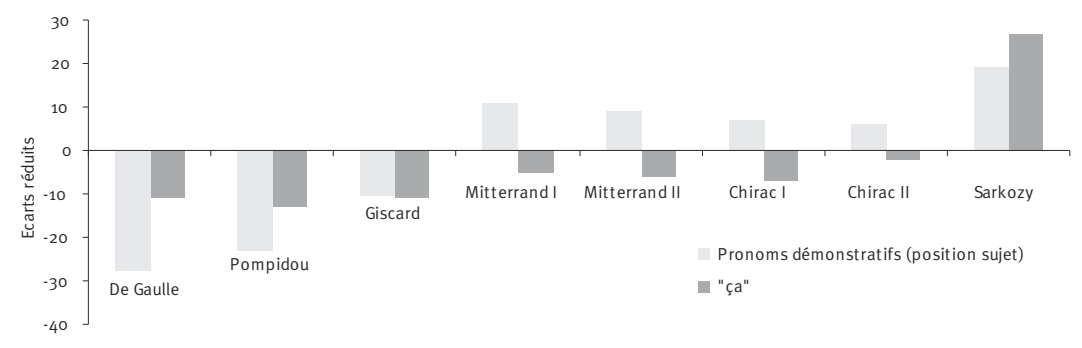

Figure 1. Distribution des pronoms démonstratifs en position sujet et de «ça » dans le corpus présidentiel (1958-2012). Calcul des spécificités [Hyperbase 9.0-2013]

Les «ça ne peut plus durer», «ça suffit comme ça », «ce n'est plus possible», «ce n'est pas vrai», "c'est insupportable» se multiplient dans le discours jusqu'à saturer le corpus et à constituer la première signature verbale du sarkozysme versus les autres présidents. Et une comparaison avec ses contemporains ou les candidats de 2007 et 2012 confirme l'analyse.

Après une exégèse linguistique poussée proposée ailleurs (Mayaffre, à paraître), il est possible de donner ici une interprétation pragmatique ou sociolinguistique du phénomène : les ça, sans référence nette, permettent de désigner fermement sans nommer précisément; ils permettent à la fois de montrer et de sous-entendre; ils disent au fond sans dire ; montrent d'une certaine manière ce que l'on ne peut pas voir; signifient tout et n'importe quoi. Parce qu'il s'agit d'un démonstratif, le démonstratif neutre a une puissance de mons- 
tration évidente; parce qu'il s'agit d'un neutre à la référence fuyante, on ne sait ce qu'il montre ou recouvre exactement.

Ainsi la recevabilité des centaines de ce ou de ça qui émaillent le discours est fonction de la connivence entre le locuteur et l'interlocuteur qui s'entendent en deçà du mot sur ce qui est sous-entendu : le ça implique et construit une connivence entre N. Sarkozy et les téléspectateurs. Avec le ça, N. Sarkozy et le peuple communient (on s'entend sur quelque chose) avec d'autant plus de ferveur qu'on ne sait exactement sur quels motifs sourds.

En 2012, dans son meeting en plein air place de la Concorde, N. Sarkozy s'écrie en s'auto-établissant comme la voix du peuple : "Le peuple de France dit “ça suffit!” " ${ }^{8}$ Et ce passage fait écho à la campagne 2007 où le candidat s'était emporté dans de longues anaphores rhétoriques répétées dans plusieurs meetings : "ça ne peut plus durer..., ça ne peut plus durer..., ça ne peut plus durer... »9.

N. Sarkozy semble ainsi surfer sur le ras-le-bol populaire sans avoir à se montrer très précis quant au motif du mécontentement, et le peuple, quant à lui, semble avoir trouvé un porte-voix - sinon un programme politique - pour exprimer une colère confuse; de plus, comme noté par P.-A. Taguief dans la définition du populisme, cette colère confusément exprimée débouche naturellement sur l'idée d'un changement indispensable («ça suffit» ou «ça ne peut plus durer» = «tout cela doit changer »).

Ajoutons que le discours du démonstratif ressemble à une mise à l'index ou à une vindicte populaire. N. Sarkozy ne montre pas du doigt mais montre du mot (ce, cet, ceux-là, celui-ci) : mise à l'index d'autant plus virulente peutêtre, et efficace, que les choses et les personnes désignées sont non pas explicites mais sous-entendues ou doxales. Par exemple, en 2012, N. Sarkozy brocarde une à une les positions de son concurrent socialiste, et après chaque démonstration conclut dans une expression radicale : «Et ÇA veut gouverner la France / Et ÇA veut diriger la France ${ }^{10}$.

Il faut prendre au sérieux ces passages puisqu’ils sont préparés et répétés. La vulgarité du procédé doit être prise au sens étymologique. Le ça - le premier mot statistique de N. Sarkozy - est celui du vulgum pecus : dans la forme et dans le fond, il est l'agent linguistique du populisme ou de la ferveur/imitation / flatterie populaire; dans le détail, nous trouvons là l'explication de la préférence de ça sur des ceci ou cela plus littéraires.

Mais si le démonstratif neutre, dans sa forme syncopée (ça), est le plus saillant dans le discours, les démonstratifs en général se multiplient. Nous en comptons plusieurs milliers, et il devient dérisoire d'en citer quelques-uns puisque c'est l'effet de masse qui compte. Retenons entre mille exemples

8. N. Sarkozy, 15 avril 2012, La Concorde.

9. Par exemple N. Sarkozy, 23 février 2007, Perpignan.

10. N. Sarkozy, 30 mars 2012, Besançon. 
celui-ci : «Je me souviens de CEUX qui voulaient séparer les hommes et les femmes dans les piscines municipales. $»^{11}$

On ne trouve pas dans le discours les personnes précisément désignées; on devine qu'il s'agit d'une certaine classe politique (laquelle précisément?); on soupçonne la gauche; N. Sarkozy vise, semble-t-il, Martine Aubry - sinon Hollande? - dont la municipalité aurait autorisé en 2009 un créneau horaire préférentiel pour des femmes obèses. Mais la vindicte peut s'abattre sur ces ennemis du peuple; ennemis d'autant plus dangereux qu'ils semblent s'avancer masqués par le fait même du discours allusif.

En d'autres termes, les démonstratifs sont des gestes verbaux qui montrent au peuple ce qu'il veut bien voir; qui montrent l'évidence qui n'a pas besoin de s'expliciter; qui, au sens d'Aristote, embrassent les topoï ou la doxa, c'est-àdire ces idées reçues et admises qui n'ont plus besoin de se justifier et même de s'énoncer. Et dans une posture performative, celui qui est investi de ce pouvoir de montrer à tout le monde ce que chacun veut bien voir, devient de facto la voix du peuple ou le tribun de la plèbe.

\section{On : une menace indéfinie}

Si la première caractéristique du discours est la fréquence de ça, la seconde est tout aussi inattendue, et éclaire d'une même lumière l'analyse : l'emploi du pronom on. Ici aussi le traitement statistique et comparatif mis en place est éloquent (figure 2).

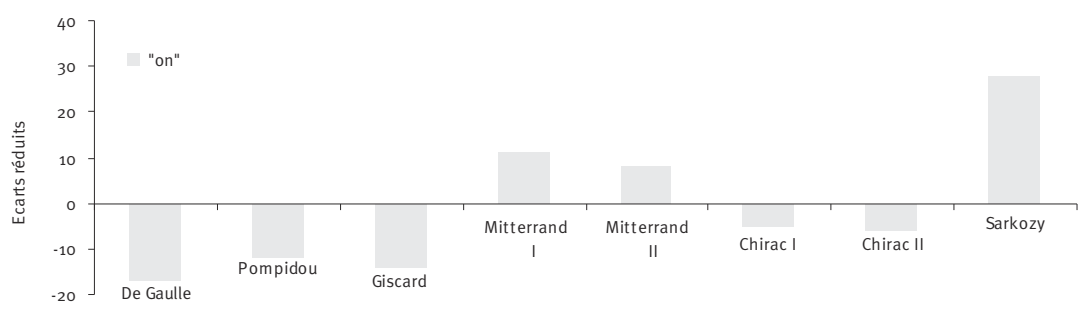

Figure 2. Distribution de on dans le corpus présidentiel (1958-2012).

Calcul des spécificités [Hyperbase 9.0-2013]

On est un des mots les plus étranges de la langue française, sans beaucoup d'équivalents dans les langues européennes. Les linguistes hésitent à considérer ce pronom surnuméraire comme singulier ou pluriel, défini ou indéfini, personnel ou impersonnel, parfois inclusif (assimilable à nous) et parfois exclusif (assimilable à vous ou à ils), etc. ${ }^{12}$

11. N. Sarkozy, 19 février, Marseille.

12. Le on a donc fait l'objet d'études nombreuses. Par exemple : Fløttum etal., 2007. 
Ainsi, il ressort, ici aussi, que pour décrypter ce «on illusionniste» (Atlani, 1984), il faut une connivence entre le locuteur et l'interlocuteur: le on, comme le ça, réclame et construit la complicité ou la communion entre le locuteur (N. Sarkozy) et l'interlocuteur (le peuple) qui s'entendent sur un non-dit, sur un ON-dit, sur un qu'en dira-t-ON.

Les «lois en faveur du travail, de l'emploi et du pouvoir d'achat» (TEPA) votées durant l'été 2007 resteront comme l'acte fondateur du quinquennat. Et dans ce TEPA, la quasi-suppression des droits de succession fut un des éléments les plus problématiques puisque, à rebours du discours de campagne, elle semblait favoriser la rente sur le travail et l'héritage sur le mérite. Dans un passage décisif, N. Sarkozy justifie la mesure devant un journaliste s'inquiétant d'une mesure fiscale favorable aux plus aisés : "C'est un cadeau aux riches? Quand ON a travaillé toute sa vie, de devoir en plus payer un impôt parce qu'ON laisse à ses enfants le produit d'une vie de travail? »13

La pertinence de l'argumentation tient tout entière dans le $o n^{14}$. Qui sont ces « on »? Tous les travailleurs pourront s'y reconnaître. Pourtant, bien avant 2007 , si ces derniers possédaient un héritage moyen ou modeste à transmettre, ils se trouvaient déjà exonérés de cette imposition progressive ${ }^{15}$. De plus, jamais les droits de succession ne portent sur le travailleur qui, décédé, serait bien en mal de payer un impôt du fond de son caveau. Ils portent sur l'héritier, ce qui n'est pas la même chose. Mais par un on incertain ou mal défini, N. Sarkozy peut toucher tout le monde, particulièrement en s'apitoyant sur le sort d'un peuple taxé par l'État même jusque dans la mort.

D’une autre manière en 2007, N. Sarkozy s'était exprimé ainsi à la télévision :

Personne n'est obligé, je répète, d'habiter en France, mais quand ON habite en France, $\mathrm{ON}$ respecte ses règles, c'est-à-dire qu'ON n'est pas polygame [...]. ON ne pratique pas l'excision sur ses filles, ON n'égorge pas le mouton dans son appartement et $\mathrm{ON}$ respecte les règles républicaines. ${ }^{16}$

Parce on, tous les Français - et même au-delà - pourront se sentir concernés, à l'exclusion de quelques-uns qui, sans être nommés, se reconnaîtront. L'art ici est d'amalgamer, par la négative, des populations hors du on. Indistinctement, les musulmans (l'Aïd-el-Kébir) ou certains Noirs africains (l'excision) sont mis à l'index. Personne n'est véritablement nommé, mais l'auditeur peut aisément entrevoir l'image de l'Étranger que N. Sarkozy lui suggère.

13. N. Sarkozy, 29 novembre 2007, interview télévisée (TF1-France 2).

14. On remarque aussi l'usage de l'interrogation qui retourne la charge du discours (voir infra).

15. Ainsi, le rapport complet du Sénat du 20 novembre 2002, sous l'autorité de Philippe Marini, établissait déjà, au début des années deux mille, que $75 \%$ des successions ne donnaient pas lieu à perception de droits. La mesure de 2007 ne concernait donc que le quartile supérieur des contribuables les plus aisés.

16. N. Sarkozy, 5 février 2007, interview télévisée (TF1, 21 heures). 
Le on est au discours populiste ce que le nous est au discours communiste : sa signature pronominale ${ }^{17}$. Là où, sous la forme d'appositions réflexives, «nous, les ouvriers, nous» ou «nous, les communistes, nous», le nous servait à construire fermement une conscience de classe ou une identité partisane, le on, indéfini, sert à sous-entendre une communauté dans laquelle chacun et tout le monde pourra se reconnaître ou s'exclure, s'impliquer ou se soustraire. On est parfois une menace indéfinie qui pèse sur un peuple en danger que N. Sarkozy défend, parfois au contraire un graal que l'électeurvoudra atteindre. Indistinctement, le on est la figure négative de l'autre ou celle d'un peuple sans visage avec qui N. Sarkozy communie à travers l'écran de télévision.

\section{Le César : construire l’autorité}

Comme il n'y a pas de populisme sans peuple, il n'y a de populisme sans un chef qui puisse prétendre mener, diriger, incarner. Beaucoup d'aspects du discours de N. Sarkozy sont ainsi tendus par l'objectif de construire un ethos supérieur ou une image charismatique. Là encore, la comparaison chiffrée avec les discours de ses prédécesseurs servira de guide.

Je veux

De tous les présidents de la République, N. Sarkozy n'est certes pas le plus narcissique. Et au bal des egos, N. Sarkozy est battu par Mitterrand qui confondit le premier la France et lui-même. Il n'empêche que le surusage du je chez N. Sarkozy reste magistral (figure 3).

L'étude s'éclaire d'abord du point précédent. Dans la plaine sans visage que construit le discours du on émerge d'autant mieux la virilité du je charismatique. Les passages sont nombreux qui jouent ainsi de la dialectique on/je:

L'authenticité se lit sur mon visage, JE ne triche pas, JE ne truque pas. [...]. ON peut être en désaccord avec ce que JE fais, ON peut ne pas M'aimer [...], c'est la démocratie, mais personne ne peut dire que J'ai menti, que J'ai travesti. [...] JE n'ai pas menti [...] et J'assume. Alors JE comprends que c'est curieux en France parce qu'ON n'assume pas quand $\mathrm{ON}$ fait partie des élites françaises en général, c'est même la marque de fabrique. ON se réfugie derrière la pensée unique, tous, comme des moutons, ON pense la même chose au même moment. MOI, J'essaye d'assumer. ${ }^{18}$

L'étude du je sarkozyste s'enrichit si l'on observe les verbes dont le pronom est le sujet. Quand chez Mitterrand le je pense et le je dis dominent, chez N. Sarkozy c’est la martialité du je veux qui s’impose (figure 3).

17. Voir nécessairement «Le nous politique », Mots, nº10, 1985.

18. N. Sarkozy, 8 janvier 2008, conférence de presse (pour la charge contre les «élites », voir infra). 


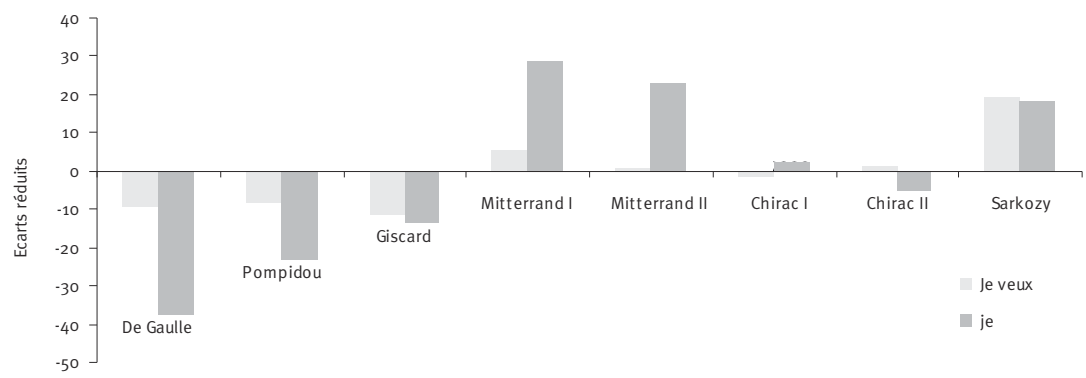

Figure 3. Distribution «Je » et «Je veux» dans le corpus présidentiel (1958-2012). Calcul des spécificités [Hyperbase 9.0-2013]

Le sarkozysme est un volontarisme verbal. L'image charismatique du chef s'exprime par l'exhibition d'un «vouloir» tout puissant; le plus souvent à l'indicatif (je veux) rarement au conditionnel (je voudrais). Le chef est celui qui veut et la volonté est l'attribut nécessaire du chef. Cette récurrence n'a échappé à personne, et, à bout d'argument, Michel Onfray n'a pour finir d'autre explication que la piste philosophique avec la «tyrannie du vouloir» de Schopenhauer, et plus encore psychanalytique avec le je veux irrésolu de la toute-puissance infantile ${ }^{19}$.

... ne...pas

Si le volontarisme au sens linguistique le plus élémentaire ( je veux) est un élément de la construction charismatique, il ne s'agit que d'une des deux facettes du discours.

L'autre facette est l'autorité : le chef c'est celui qui veut, mais tout autant celui qui ne veut pas, celui qui interdit, qui exclut, qui défend : celui qui a l'autorité pour dire «non».

Avec le ça et le on, la récurrence des signes de négation est un des traits les plus caractéristiques du discours sarkozyste versus les autres présidents ou Ségolène Royal en 2007 et François Hollande en 2012. Après examen de tous les enchaînements syntaxiques du corpus présidentiel (1958-2012), Hyperbase 20 calcule que l'enchaînement syntaxique « pronom + adverbe + verbe » est un des traits les plus remarquables du sarkozysme; et en français, cet enchaînement concerne presque exclusivement la tournure négative. Ainsi les je ne veux..., les il ne faut..., vous ne pouvez..., il n'est (pas vrai que)..., etc., se multiplient.

19. Michel Onfray, 4 février 2008, Université populaire de Caen [http://www.dailymotion.com/ video/x8hisy_841-la-tyrannie-du-vouloir-ou-sarko_news], consulté le $1^{\mathrm{er}}$ novembre 2011.

20. Hyperbase est le logiciel de logométrie utilisé pour ces études, conçu par Étienne Brunet et diffusé par le CNRS et l’Université de Nice (UMR 7320, Bases, Corpus, Langage). 
Il faut comprendre les négations - quasiment une phrase sur trois chez N. Sarkozy - dans l'intimité syntaxique d'un discours qui entend « restaurer l'autorité »; c'est le vieil ethos du ferme ministre de l'Intérieur que Sarkozy-président travaille.

La campagne électorale de 2007 donne la meilleure explication de ce discours et de ses motivations idéologiques :

Dans cette élection, il s'agit de savoir si l'héritage de Mai 68 doit être perpétué ou s'il doit être liquidé une bonne fois pour toutes.

Je veux tourner la page de Mai 68. Mais il ne faut pas faire semblant. ${ }^{21}$

Au «il est interdit d'interdire » ou au «jouir ensemble» de 1968, N. Sarkozy entend opposer un discours d'autorité ou un discours de l'Interdit; le mot autorité devenant une des grandes spécificités lexicales, comme la récurrence de l'adverbe non ou des tournures négatives ${ }^{22}$.

Et ici, il faut souligner le courage politique de N. Sarkozy. Le cœur de son électorat se trouve en effet chez les personnes âgées, au point qu'en 2007, si les seniors n'avaient pas voté, ce serait Ségolène Royal qui aurait été élue23. Aussi ce discours de et sur l'autorité, ce discours où «il est autorisé d'interdire », ce discours où il faut liquider l'héritage permissif de 1968 a convaincu, 40 ans après les événements, une génération 68 arrivée à maturité.

Leader (je veux) et père affichant sa sévérité (vous ne pouvez pas) : c'est cette image construite par le discours qui fut plébiscitée en 2007 par une France démographiquement vieillissante, et psychologiquement fatiguée par douze ans de chiraquisme.

\section{Le peuple : dénoncer les élites}

La mécanique sarkozyste est définie : un peuple, un chef, et une communion entre les deux. Dans ce cadre, sa condition de réalisation est la suppression, ou au moins la dénonciation, des corps intermédiaires susceptibles de faire écran ou médiation : aucun président, pour ne pas dire aucun responsable politique républicain ne s'était aventuré aussi loin et avec autant de virulence dans cette rhétorique.

21. N. Sarkozy, 23 février 2007 , Perpignan.

22. Pour plus de précisions, voir Mayaffre, 2007, p. 65-80.

23. Pour la sociologie électorale (ici générationnelle) de 2007 , voir par exemple " $2^{d}$ tour présidentielle 2007. Comprendre le vote des Français. Qui a voté quoi ? » [http://www.ipsos.fr/ presidentielle-2007/pdf/ssu-2eTour.pdf] (consulté le 12 septembre 2013). 


\section{Bon sens populaire contre pensée unique}

Le discours de N. Sarkozy s'édifie souvent contre un ennemi. Dans les cas les moins habiles, celui-ci peut être un bouc émissaire, comme nous l'avons vu avec le polygame ou l'exciseur, ou dans d'autres domaines avec l'assisté ou le patron-voyou, le délinquant multirécidiviste ou la haute finance. Mais plus habilement, l'adversaire est cette élite que l'on soupçonne de détourner les aspirations populaires et de bâillonner le peuple.

L'étude est facilitée parce que N. Sarkozy fait lui-même sa propre analyse pour mieux retourner la critique :

Pendant cinq ans j'ai pu mesurer la puissance des corps intermédiaires qui s'interposent entre le peuple et le sommet de l'État. Ces corps intermédiaires qui prétendent souvent parler au nom des Français et qui en vérité, souvent, confisquent la parole des Français... Je veux rendre la parole aux Français... J'ai bien entendu les cris de ceux qui pensent que se tourner vers le peuple c'est du populisme. Au fond d'eux-mêmes ils trouvent sans doute que le peuple n'est pas assez raisonnable, que le peuple n'est pas assez intelligent...24

Le passage est exemplaire, car le lecteur aura remarqué l'usage d'un «ceux» confusément explicite qui désigne à la vindicte populaire on ne sait qui exactement (sans doute les journalistes qualifiés de «parisiens », évidemment l'intelligentsia de gauche; en tout cas «ces corps intermédiaires» que l'auditeur aura charge de reconnaître). Le passage est exemplaire surtout puisqu'il frappe d'illégitimité, par principe, la critique de populisme, précisément au nom du peuple que N. Sarkozy invoque.

Parce que N. Sarkozy était en retard dans les sondages et en difficulté avec son bilan, la campagne de 2012 dont ce passage est extrait fut certes caricaturale. Cependant, l'appel au peuple de France contre les élites (la magistrature, les universitaires, les journalistes, Saint-Germain-des-Prés) est présent déjà en 2007 et constant durant la présidence.

Dès 2007 en effet, la rhétorique est stabilisée, devant une foule enthousiaste :

Vous êtes là parce que vous en avez assez du conformisme intellectuel. Vous êtes là parce que vous en avez assez de la pensée unique. Parce que vous en avez assez que l'on vous dise ce qu'il faut faire, ce qu'il faut dire, ce qu'il faut penser. Je veux vous rendre votre liberté. Votre liberté de choix. Votre liberté de parole. Votre liberté de penser. ${ }^{25}$

Et 2012 n'est, dès lors, qu'une répétition :

24. N. Sarkozy, 19 février 2012, Marseille.

25. Id., 20 mars 2007, Villebon-sur-Yvette.

Mots. Les langages du politique $\mathrm{n}^{\circ} 103$ novembre $2013 \bullet 83$ 
Je veux être le candidat du peuple de France, Je ne serai pas le candidat d'une petite élite contre le peuple. ${ }^{26}$

Au-delà des discours de campagne, on retrouve le propos, central, par exemple dans le premier discours officiel du président en exercice :

Le 6 mai, il n'y a eu qu'un seul vainqueur, le peuple français qui ne veut pas renoncer, qui ne veut pas se laisser enfermer dans l'immobilisme et dans le conservatisme, qui ne veut plus que l'ON décide à sa place, que l'ON pense à sa place. ${ }^{27}$

N. Sarkozy s'érige ainsi en porte-parole. Il est l'expression du peuple que l'on cherche à faire taire. Il a le parler vrai des simples gens, lorsque les élites compliquent, trahissent, censurent. Et N. Sarkozy n'a de cesse de revendiquer cette parole populaire enfin libérée et décomplexée :

Vous en avez assez de la pensée unique et du politiquement correct.

Vous en avez assez que l'on ne puisse plus avoir le droit de dire qu'un voyou est un voyou. Vous en avez assez que l'on ne puisse pas parler de la nation sans être accusé de nationalisme. [...] Vous en avez assez que l'on ne puisse pas dénoncer la surévaluation de l'euro sans être accusé d'être anti-européen. Vous en avez assez que l'on ne puisse pas évoquer la France qui souffre sans être immédiatement taxé de populisme. [...]

Vous en avez assez et je vous comprends. Je veux en finir avec cette pensée unique qui est le point de rencontre de tous les renoncements, de tous les sectarismes, de toutes les arrogances. ${ }^{28}$

\section{L'interrogation}

La dénonciation d'une élite qui confisque le pouvoir d'un peuple que précisément N. Sarkozy veut incarner prend un tour particulier et systématique que la statistique textuelle repère : une multiplication des interrogations rhétoriques, sans comparaison depuis 50 ans (figure 4).

Par interrogations rhétoriques, il faut entendre les questions que N. Sarkozy se pose à lui-même, ou semble poser aux journalistes qui l'interviewent, et qui n’appellent pas de réponse.

Nous avons défini ailleurs les quatre fonctions de l'interrogation sarkozyste (Mayaffre, 2012a, p. 236-262), mais il faut développerici sa méta-fonction principale.

En posant incessamment des questions, le parler de N. Sarkozy retourne la charge du discours présidentiel. D’interrogé, N. Sarkozy devient interrogateur. D'avocat de sa politique, il devient procureur d'une autre politique (celle de la gauche jospinienne ou mitterrandienne par exemple, celle des élites).

26. Id., 19 février 2012, Marseille.

27. Id., 16 mai 2007, cérémonie d'investiture.

28. Id., 13 avril 2007, Meaux. 


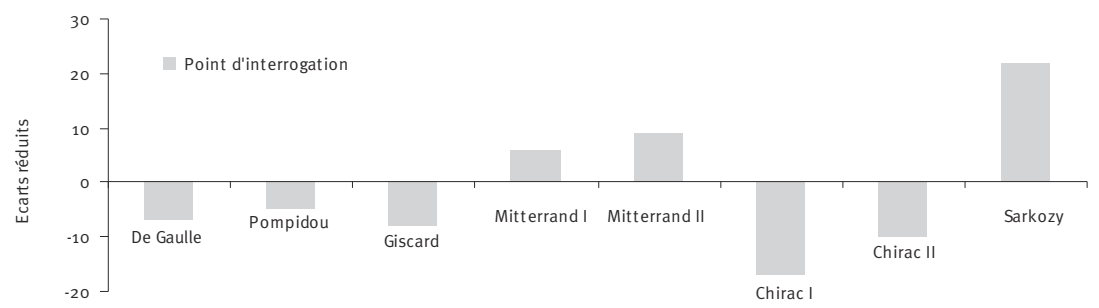

Figure 4. Distribution du point d'interrogation dans le corpus présidentiel (1958-2012). Calcul des spécificités [Hyperbase 9.0-2013]

Et à chaque question des journalistes, on comprend qu'il peut répondre par une contre-interrogation. Entre autres exemples, puisqu'on dénombre des milliers d'interrogations dans le corpus, N. Sarkozy contre-attaque comme suit sur l'affaire Woerth-Bettencourt et la mise sur écoute d'un journaliste du Monde:

Allez plus loin dans votre question, vous imaginez que c'est moi qui ai organisé le cambriolage d'un ordinateur portable d'un de vos confrères? Est-ce que c'est ce que vous pensez?.... Excusez-moi, il n'y a qu'un seul cas dans l'histoire française où un président de la République a fait écouter les journalistes. Ce n'est pas moi comme vous le savez. Il est à gauche. Oui ou non? Oui ou non ?29

Tout se passe comme si, là où habituellement le président était obligé de rendre des comptes à l'opinion publique via les questions des journalistes, c'était désormais lui qui, se réclamant du peuple, exigeait des journalistes en présence, et à travers eux de l'intelligentsia de gauche, de répondre à ses accusations. Techniquement, la stratégie est gagnante car les journalistes ne sont pas habilités à répondre. Fondamentalement surtout se joue l'essentiel : élu d'un peuple trahi par ses élites, seul porte-voix d'un peuple outragé (outragé notamment par le fait que l'on puisse soupçonner son premier représentant), c'est N. Sarkozy qui possède la légitimité pour contraindre les corps intermédiaires (ici les journalistes) à demander grâce.

Le caractère présidentiel de la Ve République, que Max Weber qualifierait sans doute de régime charismatique ${ }^{\circ}$, et le développement des moyens de gouvernement direct comme peut l'être la télévision, favorisent l'expression populiste de notre vie politique.

29. Id., 16 octobre 2010, interview télévisée (TF1 - France 2 - Canal +).

30. Voir par exemple Morel, 2010. 
Élu au suffrage universel, sans opposition directe, en dialogue immédiat avec les Français par le biais d'interviews, renseigné par des sondages sur l'état de l'opinion publique, le président peut prétendre incarner le peuple. Sans forcer le trait, et pour reprendre l'idée de charisma, nous pouvons dire que depuis la réforme de 1962, il est l’Élu.

Le discours présidentiel s'en trouve changé. Longtemps référentiel (véhicule d'un message), il devient avant tout phatique : sa fonction est de construire ce lien fusionnel entre le chef et les Français. Sa nature linguistique s'en trouve modifiée. D'abord de nature nominale dans les années soixante/soixante-dix, il devient pronominal ou adverbial. Les verbes énonciatifs ou modaux conjugués à la première personne - je pense, je vous dis, je veux - remplacent statistiquement les noms, les concepts, les idées ${ }^{31}$. Avec N. Sarkozy, d'autres stratégies de communion discursive et plébiscitaire se mettent aussi en place, tel le pronom démonstratif neutre (ça) ou le on indéfini qui disent au peuple ce qu'il veut bien entendre, et que personne avant N. Sarkozy n'avait utilisé dans de telles proportions.

«Peuple de France entend mon appel!»32: dans une geste gaullienne hors de propos - car nous n'étions plus en période de guerre -, N. Sarkozy a poussé jusqu'au bout en 2012 une rhétorique populiste pour essayer de se faire réélire. Mais au-delà du Sarkozy de 2012, ce type d'appel au peuple fait écho au sigle UMP (Union pour un mouvement populaire) qui lui-même résonne, par exemple, en Italie, avec le rassemblement berlusconien Le Peuple de la Liberté, ou à l'échelle européenne avec le Parti populaire européen (PPE). Évidemment, l'appel au peuple n'est ni nouveau dans l'histoire, ni l'apanage de la droite. Mais souvent rassemblée autour des mots république, France, démocratie (UNR, UDR, RPR, UDF, MODEM, UDI, etc.), la droite française, renouant avec les accents bonapartistes d'un RPF (Rassemblement du peuple français) par exemple, se revendique désormais moins des institutions (l’armée, l'Église, la Justice...) ou des élites (politiques, économiques, religieuses) que directement du peuple.

Cependant, si N. Sarkozy a usé d'une rhétorique aux traits populistes, force est de constater qu'il n'a réussi ni à se faire aimer ni à se faire réélire. Sondage après sondage, élection après élection, jusqu'à la perte historique du Sénat, le désamour entre le président et les Français fut plusieurs fois consommé durant le quinquennat. Parfois la crue réalité de la situation économique comme les crises qui secouèrent le monde à partir de 2008 , la montée du chômage ou l'explosion sans précédent de la dette, et la froide signification du programme politique tel l'emblématique bouclier fiscal dont devaient bénéficier non pas la plèbe mais les plus fortunés, se révèlent plus importantes aux yeux du peuple

31. Cette brève mise en perspective chronologique reprend la conclusion principale de notre ouvrage : Le discours présidentiel sous la Ve République, 2012b, op. cit.

32. N. N. Sarkozy, 15 avril 2012, La Concorde. Voir aussi la «Lettre de Nicolas Sarkozy au peuple de France» (avril 2012). 
que les techniques rhétoriques de gouvernement. La performativité du langage politique a ses limites : dire qu'on incarne le peuple ne suffit pas à l'incarner réellement, et les accents populistes du discours ne suffisent pas toujours au leader pour être populaire33.

\section{Références}

AtLANı Françoise, 1984, "On l'illusionniste », La Langue au ras du texte, Atlani et al. éd., Lille, Presses universitaires de Lille, p.13-29.

Charaudeau Patrick, 2007, Entre populisme et peopolisme. Comment Sarkozya gagné!, Paris, Vuibert.

Collovald Annie, 2004, Le "populisme du FN», un dangereux contresens, Broissieux, Le Croquant.

Esprit, 2007, n³39, «Qu'est-ce que le sarkozysme?»

Fløтtum Kjersti et al., 2007, On. Pronom à facettes, Bruxelles, De Boeck.

Guilhaumou Jacques, 2006, Discours et événement. L'histoire langagière des concepts, Besançon, Presses universitaires de Franche-Comté.

MAYAFFRE Damon, 2007, «Vocabulaire et discours électoral de Sarkozy. Entre modernité et pétainisme », La Pensée, n0352, octobre-décembre, p. 65-80, Hal-Shs [http://hal. archives-ouvertes.fr/hal-00551355/fr/], consulté le 16 mai 2013.

- 2012a, Mesure et démesure du discours. Nicolas Sarkozy (2007-2012), Paris, Presses de Sciences Po.

- 2012b, Le discours présidentiel sous la Ve République, Paris, Presses de Sciences Po.

- 2013 (à paraître), « “Ça ne peut plus durer!” La fausse opposition quantitatif/qualitatif à l'épreuve du discours sarkozyste», Corela.

Mény Yves, SuREl Yves, 2000, Par le peuple, pour le peuple. Le populisme et les démocraties, Paris, Fayard.

MOREL Laurence, 2010, «La Cinquième République, le référendum et la démocratie plébiscitaire de Max Weber», Jus Politicum, $\mathrm{n}^{\circ} 4$.

Mots, 1985, nº10, «Le nous politique».

Musso Pierre, 2011, Sarkoberlusconisme. La crise finale?, La Tour d'Aigues, L'Aube.

RASTIER François, 2011, La Mesure et le Grain, Paris, Champion.

TAguieff Pierre-André, 2007, L'illusion populiste. Essai sur les démagogies de l'âge démocratique, Paris, Flammarion.

33. Ainsi, en $2007,46 \%$ des ouvriers seulement votèrent pour Nicolas Sarkozy (contre $53 \%$ des électeurs $=-7$ ) et en $2012,42 \%$ (contre $49 \%$ des électeurs $=-7$ ). En 2007 et $2012,49 \%(-4)$ et $44 \%$ $(-5)$ des employés votèrent pour le candidat de droite. La grille des revenus est plus spectaculaire encore : en 2012, seule la tranche des «3000 euros mensuels et + » vota majoritairement pour Nicolas Sarkozy (pour la sociologie électorale de 2012, voir l'étude complète sur World Elections [http://welections.wordpress.com/2012/05/], consulté le 16 mai 2013. 